\title{
NONLINEAR VACANCY WAVES IN SOLID ${ }^{4} \mathrm{He}$
}

\author{
N. Giorgadze ${ }^{\dagger}$, R. Khomeriki ${ }^{\dagger \dagger}$ \\ ${ }^{\dagger}$ Institute of Physics, Tamarashvili Str. 6, Tbilisi 380077, Georgia \\ ${ }^{\dagger \dagger}$ Dept. of Physics, Tbilisi State University, Chavchavadze ave. 3, Tbilisi 380028, Georgia \\ E-mail: tech2000@caucasus.net,fax: (99532)304480
}

(Received March 8, 1998)

\begin{abstract}
The solitary plane wave form collective motion of defects in quantum crystals (particularly vacancies soliton in solid ${ }^{4} \mathrm{He}$ ) is investigated. The parameters specifying such solitons are calculated. It is mentioned that under specific conditions obtained here the solitons of defects can be observed experimentally.
\end{abstract}

Key words: solid helium, weakly nonlinear soliton of vacancies.

PACS number(s): 67.80.-s, 67.80.Mg

Different mobilities in quantum crystals [1] caused by the tunnelling of admixture defects (such as vacancies in solid ${ }^{3} \mathrm{He}$ or ${ }^{4} \mathrm{He},{ }^{3} \mathrm{He}$ atoms in solid ${ }^{4} \mathrm{He}$ or vice versa) have been the subject of intensive studies during a long time (particularly, quantum diffusion phenomena [2], spin-lattice relaxation in solid ${ }^{3} \mathrm{He}$ [3] and solid Hydrogen [4] caused by tunnelling effects etc.). These mobilities can be described by a fictitious spin formalism [1]. Thereby the existence of linear wave-like excitations of defects (in case of their small concentration) can be also supposed [5,1], though the experimental verification of the latter assertion is connected with some problems: The observation of different manifestations of the wave nature of vacancies is almost impossible yet because of their negligible concentration [3].

On the other hand, any instability may cause the appearance of solitary wave of defects which can propagate within a sample with the amplitude sufficient to be observed experimentally. Therefore we study the possibility of the existence of such nonlinear waves and investigate the properties of wave excitations.

The method of the inverse scattering problem [6] is usually used to find out the exact solitary solutions of the corresponding motion equations in simple spin structures (such as ferromagnets [7], easy axis type antiferromagnets $[8]$ etc.). So it can be applied for our needs: to describe the nonlinear transition of defects by the use of the fictitious spin formalism. But as the amplitude of solitary wave (the local relative concentration of defects) should be much less than unity (otherwise at low temperatures defects are localized [2]) it is easier to apply the reductive perturbation method developed in [9].

This method has been successfully used for describing solitary waves' propagation in plasma [10] and magnetic materials [11]. Moreover, the above method can be extended for the investigation of propagation of dynamical solitons formed by short wavelength (by order of lattice parameter scale) excitations to be demonstrated below.

Using fictitious spin formalism the Hamiltonian of the system with defects can be written in the following form [1]:

$$
\mathcal{H}=\omega_{0} \sum_{f}^{N}\left(S_{f}^{z}+1 / 2\right)-\sum_{f, g}^{N} A_{f g} S_{f}^{+} S_{g}^{-},
$$

where $\mathbf{S}_{f}$ is the operator of a fictitious spin situated in the lattice site $f ; S_{f}^{z}=1 / 2$ and $S_{f}^{z}=-1 / 2$ correspond to the absence and existence of a defect in the lattice site $f$, respectively; $S_{f}^{ \pm} \equiv S_{f}^{x} \pm i S_{f}^{y} ; A_{f g}$ is a constant characterizing tunnelling of a defect from site $f$ to $g$.

The motion equation for the considered fictitious spinsystem has the form:

$$
\begin{aligned}
& \frac{d S_{f}^{ \pm}}{d t}= \pm i\left(\omega_{0} S_{f}^{ \pm}+2 \sum_{g}^{N} A_{f g} S_{f}^{z} S_{g}^{ \pm}\right), \\
& \frac{d S_{f}^{z}}{d t}=-i \sum_{g}^{N} A_{f g}\left(S_{f}^{-} S_{g}^{+}-S_{f}^{+} S_{g}^{-}\right) .
\end{aligned}
$$

Denoting $M_{f}^{ \pm} \equiv\left\langle S_{f}^{\mp}\right\rangle, M_{f}^{z} \equiv\left\langle S_{f}^{z}\right\rangle$ (the brackets $\langle\ldots\rangle$ express quantum-statistical averaging) let us determine a reduced dynamical part of $\mathbf{M}_{f}$ as $\mathbf{m}_{f} \equiv$ $\left(\mathbf{M}_{f}-\mathbf{M}_{0}\right) /\left|M_{0}\right|$ where $\mathbf{M}_{0}=M_{0} \mathbf{e}_{z}, \mathbf{e}_{z}$ is a unit vector along the $z$ axis in a fictitious space, $M_{0} \simeq-1 / 2$ and $\mathbf{M}_{0}$ is a statical value of $\mathbf{M}_{f}$. It should be noticed that using the above definitions a relative concentration of defects can be presented as

$x=x_{0}+\delta x, \quad x_{0}=M_{0}+1 / 2, \quad \delta x=\left|M_{0}\right| m_{z}$

( $x_{0}$ is a statical relative concentration of defects). Afterwards in Weiss field approximation [1] (neglecting quantum correlations e.g. $\left\langle S_{f}^{+} S_{g}^{-}\right\rangle \equiv\left\langle S_{f}^{+}\right\rangle\left\langle S_{g}^{-}\right\rangle$etc., where $f \neq g$ ), we get from $(2)$ the equation 


$$
\begin{aligned}
\frac{d m_{f}^{ \pm}}{d t}= & \mp i\left(\omega_{0} m_{f}^{ \pm}-2\left|M_{0}\right| \sum_{g}^{N} A_{f g} m_{g}^{ \pm}\right) \\
& \mp 2 i\left|M_{0}\right| m_{f}^{z} \sum_{g}^{N} A_{f g} m_{g}^{ \pm}
\end{aligned}
$$

and the relation

$$
m_{f}^{z}=\frac{1}{2}\left(m_{f}^{+} m_{f}^{-}+\left(m_{f}^{z}\right)^{2}\right)
$$

derived from the conservation law $\left|\mathbf{M}_{f}\right|^{2}=M_{0}^{2}$, which can be easily obtained averaging quantum-statistically set of equations (2) in Weiss field approximation.

Following [9] and considering the weakly nonlinear fictitious spin excitations let us search for the solution of the system of equations (4), (5) in the form

$$
\begin{aligned}
& m_{g}^{ \pm}=\sum_{\alpha=1}^{\infty} \varepsilon^{\alpha} \sum_{l=-\infty}^{\infty} m_{l}^{ \pm(\alpha)}\left(\xi_{g}, \tau\right) \cdot e^{i l\left(\mathbf{k r}_{g}-\omega t\right)} \\
& m_{g}^{z}=\sum_{\alpha=1}^{\infty} \varepsilon^{\alpha} \sum_{l=-\infty}^{\infty} m_{l}^{z(\alpha)}\left(\xi_{g}, \tau\right) \cdot e^{i l\left(\mathbf{k r}_{g}-\omega t\right)}
\end{aligned}
$$

where

$$
\begin{aligned}
& m_{-l}^{-(\alpha)}(\xi, \tau)=\left(m_{l}^{+(\alpha)}(\xi, \tau)\right)^{*} \\
& m_{-l}^{z(\alpha)}(\xi, \tau)=\left(m_{l}^{z(\alpha)}(\xi, \tau)\right)^{*}
\end{aligned}
$$

and

$$
\xi_{g}=\varepsilon\left(\boldsymbol{\kappa} \mathbf{r}_{g}-\lambda t\right), \quad \tau=\varepsilon^{2} t
$$

are slowly varying space-time variables $[9](\boldsymbol{\kappa} \equiv \mathbf{k} / k$ so the modulation along $\mathbf{k}$ is examined); $\lambda$ is the propagation velocity of modulated wave and $\varepsilon$ is a formal small parameter. $\varepsilon$ will enter (as we will see below) in combination with $\mathbf{m}$ which will play the role of the expansion parameter and in the final results we will set $\varepsilon$ to unity. As components of $\mathbf{m}_{l}\left(\xi_{g}, \tau\right)$ depend only on slow variables (7) we can suppose that their inhomogeneous parameter $\Lambda \gg a(a$ is a distance between the nearest neighboring sites of the lattice), so $\mathbf{m}_{l}\left(\xi_{g}, \tau\right)$ can be considered as a continuous function. Let us note that the indices $f$ and $g$ numerate lattice sites while the index $l$ specifies different harmonics of $\mathbf{m}_{f}$.

For calculating the combination $\sum_{g}^{N} A_{f g} m_{g}^{ \pm}$existing in equations (4) let us expand $m_{l}^{ \pm(\alpha)}\left(\xi_{g}, \tau\right)$ in the vicinity of $\xi_{f}$ since the tunnelling radius is by the order of the lattice parameter scale (so the above sum is restricted to the nearest neighborhoods) and the inhomogeneous scale of $m_{l}^{ \pm(\alpha)}(\xi, \tau)$ is supposed to be much larger than $a$. Thereby we get

$$
\begin{aligned}
& \sum_{g}^{N} A_{f g} m_{g}^{ \pm}=\sum_{l=-\infty}^{\infty} e^{i l\left(\mathbf{k r}_{f}-\omega t\right)} \sum_{\alpha=1}^{\infty} \sum_{n=0}^{\infty} \frac{(-i)^{n}}{n !} \varepsilon^{\alpha+n} \\
& \times\left\{\left(\kappa \frac{\partial}{\partial \mathbf{q}} \frac{\partial}{\partial \xi_{f}}\right)^{n}\left(A(\mathbf{q}) m_{l}^{ \pm(\alpha)}\left(\xi_{f}, \tau\right)\right)\right\}_{\mathbf{q}=l \mathbf{k}},
\end{aligned}
$$

where

$$
A(\mathbf{q}) \equiv \sum_{g}^{N} A_{f g} e^{i \mathbf{q}\left(\mathbf{r}_{g}-\mathbf{r}_{f}\right)}
$$

Substituting expressions (7), (8) into equations (4), (5) and equating the coefficients of various powers of $\varepsilon$ in the same harmonics to zero one can obtain the closed system of equations to calculate the values of $m_{l}^{ \pm(\alpha)}(\xi, \tau)$ and $m_{l}^{z(\alpha)}(\xi, \tau)$. Applying the reductive perturbation method [9] we get finally (in the third order $\varepsilon$ approximation) the nonlinear Schrödinger equation for $m_{1}^{+(1)}(\xi, \tau)$. Its well-known solution yields (in view of (3) and (5)) the expression for a relative concentration of defects

$$
\begin{aligned}
& x=x_{0}+\delta x \\
& \delta x=\delta x_{m} \cdot \operatorname{sech}^{2}\left\{\sqrt{\frac{2 \delta x_{m} \Delta}{\omega^{\prime \prime}}}(\boldsymbol{\kappa r}-\lambda t)\right\}
\end{aligned}
$$

Here it was taken into account that $\left|M_{0}\right| \simeq 1 / 2 ; \delta x_{m}$ is amplitude of $\delta x$. So the width of soliton of defects is

$$
\Lambda=\sqrt{\frac{\omega^{\prime \prime}}{2 \delta x_{m} \Delta}},
$$

where $(a, b=x, y, z)$

$$
\begin{aligned}
& \lambda=-2\left|M_{0}\right| \frac{\partial A(\mathbf{k})}{\partial \mathbf{k}} \boldsymbol{\kappa}, \\
& \omega^{\prime \prime}=-2\left|M_{0}\right| \sum_{a, b} \frac{\partial^{2} A(\mathbf{k})}{\partial k_{a} \partial k_{b}} \kappa_{a} \kappa_{b}, \\
& \Delta=-2\left|M_{0}\right| A(\mathbf{k}) .
\end{aligned}
$$

It should be mentioned that solution (11) is valid till Lighthill condition [12] $\omega^{\prime \prime} \Delta>0$ holds.

The value of $\delta x$ can be much more than statical relative concentration $x_{0}$ (weak nonlinearity requires only satisfaction of condition $\left.\delta x_{m} \ll 1\right)$.

All parameters specifying the soliton depend upon the function $A(\mathbf{k})$ (see expressions (12) and (11)). We will determine it for the bcc phase of solid Helium crystal.

Directing the axis of frame of references along the lattice edges (crystallographic axis), supposing that a quantum tunnelling is restricted to the only 8 neighboring 
sites and denoting $A_{f g}=A$ we get from definition (9)

$$
A(\mathbf{k})=8 A \cdot \cos \frac{a k_{x}}{\sqrt{3}} \cos \frac{a k_{y}}{\sqrt{3}} \cos \frac{a k_{z}}{\sqrt{3}} .
$$

Considering large wavelength excitations $k a \ll 1$ we easily obtain

$$
A(\mathbf{k})=8 A\left(1-\frac{1}{6}(a k)^{2}+\cdots\right)
$$

and thereby we get the dispersion law coinciding with the respective one discussed in [3,5]. From (12) and taking into account (14) it can be concluded that the Lighthill condition is not satisfied and consequently the existence of a solitary creation of defects is impossible for the large wavelength excitations. Analyzing further (14) we also obtain that a solitary solution does not exist if $\mathbf{k}$ is directed along the crystallographic axis $x, y, z$. But it is easy to show that in the wide range of $\mathbf{k}$ soliton solution may exist. Indeed if we examine for simplicity the case when $\mathbf{k}$ is situated in the $x y$ plane, in view of (11), (12) and (13) the following expression for the width of soliton can be obtained:

$$
\Lambda=\frac{a}{3 \sqrt{\delta x_{m}}} \sqrt{\kappa_{x} \kappa_{y} \operatorname{tg} \frac{a k_{x}}{\sqrt{3}} \operatorname{tg} \frac{a k_{y}}{\sqrt{3}}-1 / 2}
$$

Which is valid if

$$
\operatorname{tg}\left(a k_{x} / \sqrt{3}\right) \operatorname{tg}\left(a k_{y} / \sqrt{3}\right)>(1 / 2) \kappa_{x} \kappa_{y}
$$

From (15), (11) it follows that generally

$$
\Lambda \sim a / \sqrt{\delta x_{m}}
$$

and we should check that the condition $\Lambda \gg a$ should be satisfied. Furthermore as it is easy to see from (15) the width of soliton grows rapidly if $\cos \left(a k_{x} / \sqrt{3}\right) \rightarrow 0, k_{y} \neq$ 0 or $\cos \left(a k_{y} / \sqrt{3}\right) \rightarrow 0, \quad k_{x} \neq 0$.

In contrast to the linear case (when relative concentration of defects remains constant) in the case when solitary solution exists $x$ varies according to expression (11). So it can be directly observed experimentally applying $X$-ray diffraction technique. As mentioned above the vacancy soliton propagating along crystallographic axes does not exist. However the solitary collective motion can be observed e.g. if $k_{z}=0, k_{x}=k_{y}$. Then in view of (15) we get

$$
\frac{\pi}{4}<\left|\frac{a k}{\sqrt{6}}\right|<\frac{3 \pi}{4}
$$

Thus if the vacancies with such a wave number are excited (e.g. using acoustic waves) the collective motion along the 110 axis can be observed.
[1] A. Abragam, M. Goldman, Nuclear Magnetism: Order and Disorder (Clarendon Press, Oxford, 1982).

[2] A. F. Andreev, Usp. Fiz. Nauk 118, 251 (1976); D. I. Pushkarov, Zh. Eksp. Teor. Fiz. 68, 1471 (1975).

[3] N. Sullivan, G. Deville, A. Landesman, Phys. Rev. B 11, 1858 (1975).

[4] D. Zhou, M. Rall, J. P. Brison, N. S. Sullivan, Phys. Rev. B 42, 1929 (1990).

[5] J. H. Hetherington, Phys. Rev. 176, 231 (1968); A. F. Andreev, I. M. Lifshitz, Zh. Eksp. Teor. Fiz. 56, 2057 (1969).

[6] A. C. Newell, Solitons in Mathematics and Physics (Society for Industrial and Applied Mathematics, Arizona,
$1985)$.

[7] V. E. Zakharov, A. B. Shabat, Zh. Eksp. Teor. Fiz. 61, 118 (1971); M. Lakshmanan, Phys. Lett. A 61, 53 (1977).

[8] I. V. Bariakhtar, B. A. Ivanov, Solid State Commun. 34, 545 (1980).

[9] T. Taniuti, Suppl. Progr. Theor. Phys. 55, 1 (1974).

[10] T. Kakutani, Suppl. Progr. Theor. Phys. 55, 97 1974; M. Kako, Suppl. Progr. Theor. Phys. 55, 120 (1974).

[11] N. Giorgadze, R. Khomeriki, Phys. Status Solidi B 201, 235 (1997).

[12] V. I. Karpman, Nielinieinyie Volny v Dispiergiruiuschikh Sriedakh (Nonlinear Waves in Dispergive Media) (Nauka, Moscow, 1976). 